\title{
Phase-locked sustainment of photorefractive holograms using phase conjugation
}

\author{
Yong Qiao, Demetri Psaltis, Claire Gu, ${ }^{\text {a) John Hong, a) }}$ \\ Pochi Yeh, ${ }^{\text {b) }}$ and R. R. Neurgaonkara) \\ Department of Electrical Engineering, California Institute of Technology, Pasadena, California 91125
}

(Received 19 April 1991; accepted for publication 22 July 1991)

\begin{abstract}
A method for sustaining multiply exposed photorefractive holograms, in a phase-locked fashion, by using a pair of phase-conjugating mirrors is described. It is shown that a steady state exists where the overall diffraction efficiency is independent of the number of holographic exposures and the final holograms are exactly in phase with the initial ones. Both analytical and experimental results are presented.
\end{abstract}

In this communication, we describe a system in which a multiply exposed photorefractive hologram can reach a steady state with overall efficiency independent of the number of holographic exposures $M$. Previously reported exposure schedules ${ }^{1,2}$ for such multiply exposed holograms result in a diffraction efficiency of the individual holograms proportional to $M^{-2}$, while diffraction efficiency of the composite hologram scales as $M^{-1}$ if the individual exposures are statistically independent. Furthermore, the phases of the recorded holograms in this system remain locked, which is not true for a previously reported copying method. ${ }^{3}$ The system diagram is shown in Fig. 1, where the primary hologram is complemented by two phase-conjugating mirrors (PCMs). In this system, the PCMs are photorefractive crystals in the four-wave mixing configuration. They must share the same pair of pump beams so that the phase-conjugate beams retain the same relative phase. The basic idea of this system is to record a primary hologram with external beams, read out this primary hologram with the reference beam $o_{\text {, }}$, and finally copy the hologram that is read out back to the same crystal using the two PCMs. For photorefractive holograms produced only by diffusion, there is a phase shift of $\pi / 2$ between the interference pattern and the corresponding hologram. When the reference beam $o_{j}$ is on and if the crystal axis is oriented properly, the interference pattern formed by the reference beam $o_{j}$ and the diffracted beam $t_{i}$ will create a hologram that is exactly in phase with the original hologram. ${ }^{4}$ When these two beams are phase conjugated (to produce the beams $o_{j}^{\prime}$ and $\left.t_{i}^{\prime}\right)$, the hologram that the phase conjugate beams create is exactly in phase with the original hologram, and therefore the latter gets enhanced and sustained..

We assume that the hologram is recorded with a planewave reference beam whose angle is selected from one of several possible positions. An arbitrary signal beam can also be decomposed into a set of plane-wave components. The hologram can then be described as a superposition of gratings, each being the result of the interference between the $j$ th reference beam and the $i$ th plane-wave component

\footnotetext{
a) Permanent address: Rockwell International Science Center, Thousand Oaks, California 91360.

b) Permanent address: Department of Electrical and Computer Engineering, University of California, Santa Barbara, California 93106.
}

of the signal beam. Let $E_{i j, 1}$ denote the amplitude of the space-charge field recorded in the photorefractive crystal that corresponds to the $(i j)$ th grating. Using the fact that the hologram is phase locked in this system, the first-order dynamic equation describing the formation and decay of hologram can be written as ${ }^{5}$

$$
\tau_{t} d\left|E_{i j, 1}\right| / d t=-\left|E_{i j, 1}\right|+m_{i j}\left|E_{s}\right| .
$$

In Eq. (1), $m_{i j}$ is the modulation depth of the interference pattern, given by

$$
m_{i j}=2 t_{i}^{\prime} o_{j}^{\prime} / I_{0}
$$

where $I_{0}$ is the total illuminating intensity; $\tau_{t}$ is the characteristic time constant, which can be written as

$$
\tau_{t}=\tau_{t}^{\prime} / I_{0}
$$

$\tau_{t}^{\prime}$ and $\left|E_{s}\right|$ are real parameters that depend upon the crystal properties and the recording geometry. The amplitude diffraction efficiency of the $(i j)$ th grating is denoted by $w_{i j}$ and it is related to the space-charge field by

$$
w_{i j}=\sin \left(\beta\left|E_{i j, 1}\right|\right),
$$

where $\beta$ depends on the effective electro-optic coefficient of the crystal, the hologram thickness, and the recording wavelength.

If we define

$$
y_{i j}=\beta\left|E_{i j, 1}\right|
$$

and

$$
c=2 \beta\left|E_{s}\right|,
$$

then a set of simplified equations is obtained:

$$
\begin{aligned}
& \frac{d y_{i j}}{d t}=\frac{1}{\tau_{t}^{\prime}}\left(-I_{0} y_{i j}+c t_{i}^{\prime} o_{j}^{\prime}\right), \\
& w_{i j}=\sin \left(y_{i j}\right),
\end{aligned}
$$

where we have used Eqs. (2) and (3).

We first consider the case of single reference beam with $N$ gratings recorded in the crystal. With the reference beam on (see Fig. 1 for illustration), the dynamics of the PCM system are described by Eqs. (7) and (8), with

$$
t_{i}^{\prime}=A r_{1} w_{i j}
$$




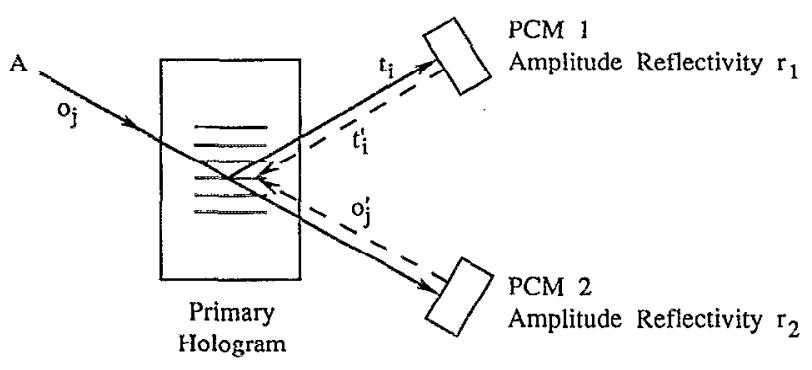

FIG. 1. Schematic diagram for the hologram sustaining system with a single reference beam.

$$
\begin{aligned}
& o_{j}^{\prime}=A r_{2}\left(1-\sum_{k=1}^{N} w_{k j}^{2}\right)^{1 / 2}, \\
& I_{0}=A^{2}+A^{2} r_{1}^{2} \sum_{k=1}^{N} w_{k j}^{2}+A^{2}\left(1-\sum_{k=1}^{N} w_{k j}^{2}\right) r_{2}^{2}
\end{aligned}
$$

$A$ is the real amplitude of the reference beam, and $r_{1}$ and $r_{2}$ are the amplitude reflectivities of the two PCMs. Substituting Eqs. (9)-(11) into Eq. (7), we obtain

$$
\begin{aligned}
\frac{d y_{i j}}{d t}= & \frac{r_{2}^{2} A^{2}}{\tau_{t}^{\prime}}\left[-\left(a+\left(\rho^{2}-1\right) \sum_{k=1}^{N} w_{k j}^{2}\right) y_{i j}\right. \\
& \left.+c \rho w_{i j}\left(1-\sum_{k=1}^{N} w_{k j}^{2}\right)^{1 / 2}\right],
\end{aligned}
$$

where $a=1+1 / r_{2}^{2}$ and $\rho=r_{1} / r_{2}$. In deriving Eq. (12), we have assumed that $c, \tau_{t}^{\prime}$, and $r_{1}$ are all independent of the grating index $i$. This assumption is valid if the spatial bandwidth of the signal beam is small.

The steady state of the system is obtained by setting $d y_{i j} / d t=0$ in Eq. (12):

$$
y_{i j}=\frac{c \rho w_{i j} \sqrt{1-\Sigma_{k=1}^{N} w_{k j}^{2}}}{a+\left(\rho^{2}-1\right) \Sigma_{k=1}^{N} w_{k j}^{2}} .
$$

The steady-state diffraction efficiency $w_{i j}^{(s)^{2}}$ can be solved from the above equation and Eq. (8). Assuming low diffraction efficiencies, a sufficient but not necessary condition for nonzero steady state is

$$
\rho>\max \{a / c, 1\} \text {. }
$$

It can be shown, using straightforward perturbation analysis, that the steady state is stable under this condition. For typical photorefractive crystals, $c=0.2-10$. In the case of small $c$, the steady-state overall diffraction efficiency satisfies the condition $\sum_{k=1}^{N} w_{k j}^{(s)} \ll 1$, which implies that $w_{i j}^{(s)} \approx y_{i j}^{(s)}$ and $\sqrt{1-\Sigma_{k=1}^{N} w_{k j}^{(s)}} \approx 1$. The latter is actually the undepleted reference approximation. With these approximations, Eq. (13) can be solved explicitly, and it yields

$$
\sum_{k=1}^{N} w_{k j}^{(s)}=\frac{c \rho-a}{\rho^{2}-1} \equiv \eta_{1}
$$

With the approximation $w_{i j} \approx y_{i j}$, Eq. (12) also shows that all the gratings rise or decay with the same time constant,

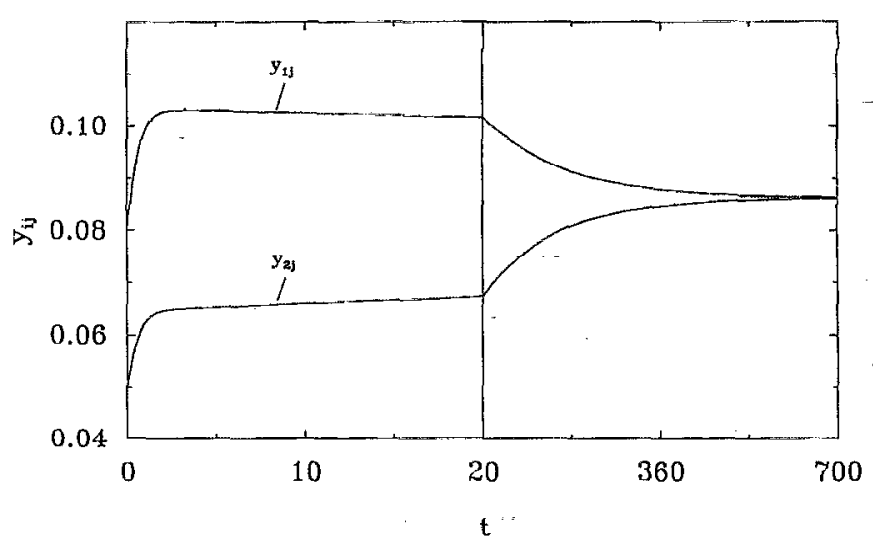

FIG. 2. Numerical simulation of Eq. (12) in which the primary hologram consists of two gratings with different initial amplitudes. The simulation was performed with the following parameters: $\rho=8, a=2, c=0.37$, $y_{1 j}(0)=0.08, y_{2 j}(0)=0.05$, and $\left(r_{2}^{2} A^{2} / \tau_{i}^{\prime}\right)=1$.

which implies that $w_{i j}^{(s)}: w_{k j}^{(s)}=w_{i j}^{(0)}: w_{k j}^{(0)}$ for any $i, k$, $1 \leqslant i, k \leqslant N$. Here $w_{i j}^{(0)}$ represents the initial value of $w_{i j}$. So Eq. (15) can be rewritten as

$$
w_{i j}^{(s)^{2}}=\eta_{1} \frac{w_{i j}^{(0)^{2}}}{\sum_{k=1}^{N} w_{k j}^{(0)^{2}}} .
$$

This property of grating strength normalization is very useful in many applications including neural network implementation $^{6-9}$ since it effectively prevents interconnection weights from either decaying or saturating. If the primary hologram is formed through a sequence of $M$ exposures using the exposure schedule of Ref. 2, then $w_{i j}^{(0)^{2}}$ $\sim M^{-2}$ for all $i j$ pairs. Therefore we can see from Eq. (16) that the steady-state diffraction efficiency $w_{i j}^{(s)^{2}}$ is independent of $M$. For large values of $c$, the above approximations do not hold and we must solve Eqs. (8) and (13) for the exact steady states.

The approximation $w_{i j} \approx y_{i j}$ used to derive the steady state takes into account only the first term in the expansion of the sine function in Eq. (8). This approximation, however, is insufficient when the overall diffraction efficiency starts approaching its steady-state value $\eta_{1}$. When that happens, $d y_{i j} / d t \approx 0$ and the higher-order terms of the sine expansion cannot be ignored in the dynamic equation (12). These higher-order terms, according to our model, have an equalizing effect that will lead the system to a final steady state where all the holographic gratings reach the same diffraction efficiency. This same steady-state diffraction efficiency can be found by solving Eqs. (8) and (13). For large $\rho$ and low diffraction efficiencies, this equalizing process occurs much slower than the grating normalization process we discussed above, so that in practice we usually observe the latter case as a quasisteady state. Shown in Fig. 2 is a numerical simulation of Eq. (12) in which the primary hologram consists of two gratings with different initial amplitudes. Initially the ratio of the strengths of the two gratings remains constant until a quasisteady state is 


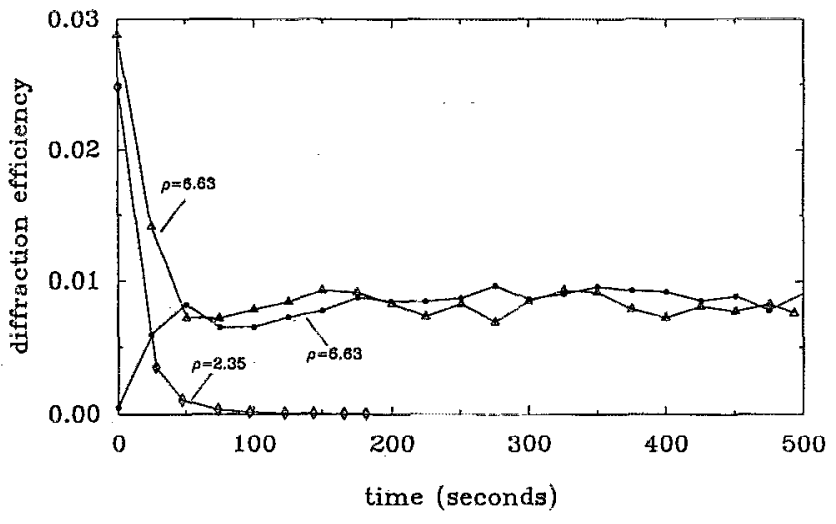

FIG. 3. Experimental results for the hologram sustaining system. $r_{2}$ $=1$ in all the experiments. For $\rho=6.63$, the same steady-state diffraction efficiency is reached when we start with either low $(\bullet)$ or high $(\Delta)$ diffraction efficiency. For $\rho=2.35$, the diffraction efficiency decays to zero $(\diamond)$.

reached. Afterwards, the strengths of the two gratings slowly converge to a common final steady-state value.

The experimental system consists of an SBN crystal as the primary hologram and a $\mathrm{BaTiO}_{3}$ crystal for the PCMs. The first experiment examines the dynamics of a single grating recorded in this system. The relevant parameters are $r_{2}=1$ and $c=0.37$. Figure 3 shows three experimental curves measuring the changes in diffraction efficiency with time. When $\rho=r_{1}=6.63$, condition (14) was satisfied and the system reached an overall steady-state diffraction effciency of about $0.845 \%$, independent of the initial condition. For comparison, the theoretical value for the steadystate diffraction efficiency is $\eta_{1}=1.06 \%$ from Eq. (15). The discrepancy between the experimental and theoretical results may be due to the wave-mixing effect in the SBN and the dependence of phase conjugate reflectivity on the probe intensity. When $\rho$ was reduced to 2.35 , however, the system did not have a nonzero steady state and thus the grating decayed to zero as predicted.

The second experiment investigates the steady-state behavior of multiple gratings recorded in the system. This was done by recording the Fourier transform hologram of an image, which consisted of multiple gratings resulting from different spatial frequency components of the Fourier transform. Figure 4(a) shows the reconstruction of the image from the SBN when it was first recorded and Fig. 4(b) shows the steady-state hologram. Although there is some distortion in the steady-state hologram, it can be seen that the grating normalization effect is dominant since all the spatial frequency components are roughly proportional to their initial conditions.

In order to store information in a volume hologram, we need multiple reference beams. For the multiple reference beam case, assuming that there are $N$ plane-wave components in the signal beam and $R$ reference beams, there are two possible ways of sustaining them. One way is to bring in the reference beams cyclically, and the other is to use mutually incoherent reference beams and have thern on simultaneously. Following derivations similar to the

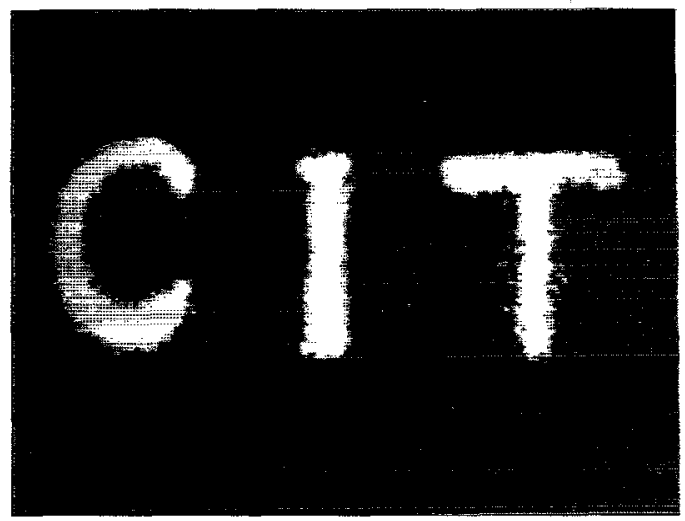

(a)

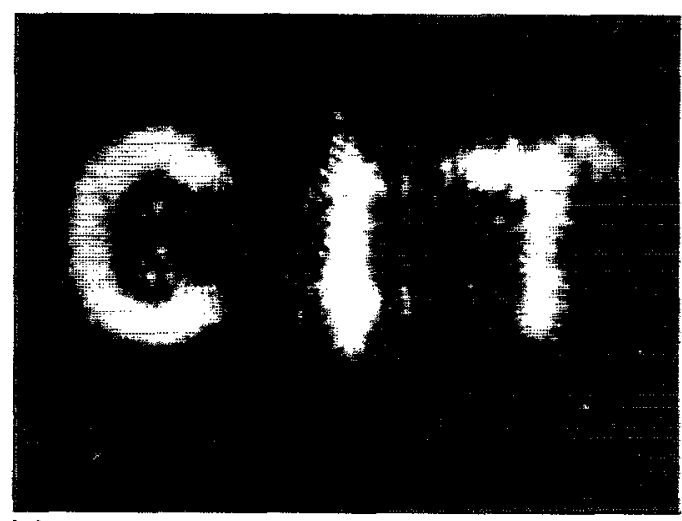

(b)

FTG. 4. (a) The reconstruction of the Fourier transform hologram of an image initially recorded in the SBN crystal. (b) The steady-state response of the hologram stored in the SBN with the initial condition being a hologram of the image shown in (a).

single reference case, it can be shown that both of these schemes lead to the same grating-normalization steady state:

$$
w_{i j}^{(s)^{2}}=\left(\frac{c \rho-R a}{\rho^{2}-1}\right) \frac{w_{i j}^{(0)^{2}}}{\Sigma_{l=1}^{R} \Sigma_{k=1}^{N} w_{k l}^{(0)^{2}}} .
$$

From Eq. (17), the number of reference beams that can be supported is bounded by

$$
R<c \rho / a=c r_{1} r_{2} /\left(1+r_{2}^{2}\right) .
$$

The authors gratefully acknowledge helpful discussions with Hsin-Yu Li: At Caltech, this work was supported by the Defense Advanced Research Projects Agency and the Air Force Office of Scientific Research.

${ }^{1}$ K. Bløtekjaer, Appi. Opt. 18, 57 (1979).

${ }^{2}$ D. Psaltis, D. Brady, and K. Wagner, Appl. Opt. 27, 1752 (1988).

${ }^{3}$ D. Brady, K. Hsu, and D. Psaltis, Opt. Lett. 15, 817 (1990).

${ }^{4}$ D. L. Staebler and J. J. Amodei, J. Appl. Phys. 43, 1042 (1972).

${ }^{5}$ N. V. Kukhtarev, V. B. Markov, S. G. Odulov, M. S. Soskin, and V. L. Vinetskii, Ferroelectrics 22, 949 (1979).

${ }^{6} D$. Psaltis, D. Brady, X. -G. Gu, and S. Lin, Nature 343, 325 (1990).

${ }^{7}$ L. S. Lee, H. M. Stoll, and M. C. Tackitt, Opt. Lett. 14, 162 (1989).

${ }^{8}$ Y. Owechko, Appl. Opt. 26, 5104 (1987).

${ }^{9}$ D. Z. Anderson and M. C. Erie, Opt. Eng. 26, 434 (1987). 